\title{
HBV Infection Promotes the Occurrence and Development of Hepatocellular Carcinoma through Impairing the Inhibitory Effect of PPP2R5A on MAPK/AKT/WNT Signaling Pathway
}

\author{
Xuejing Lin 1,2*, Ziming Mao1,3*, Qin Zhang1, Lei Chen¹, Haihua Qian¹, Chunying Liu1,2\#, \\ Changqing Su1,2\#
}

\begin{abstract}
${ }^{1}$ Department of Molecular Oncology, Eastern Hepatobiliary Surgery Hospital, Navy Military Medical University, Shanghai, China ${ }^{2}$ National Center for Liver Cancer, Navy Military Medical University, Shanghai, China

${ }^{3}$ Department of Endocrinology, Shanghai Ninth People's Hospital, Shanghai Jiao-Tong University School of Medicine, Shanghai, China Email: "suchangqing@gmail.com, "cyliu@sibcb.ac.cn
\end{abstract}

How to cite this paper: Lin, X.J., Mao, Z.M., Zhang, Q., Chen, L., Qian, H.H., Liu, C.Y. and Su, C.Q. (2021) HBV Infection Promotes the Occurrence and Development of Hepatocellular Carcinoma through Impairing the Inhibitory Effect of PPP2R5A on MAPK/AKT/WNT Signaling Pathway. Engineering, 13, 197-214.

https://doi.org/10.4236/eng.2021.134015

Received: March 9, 2021

Accepted: April 22, 2021

Published: April 25, 2021

Copyright $\odot 2021$ by author(s) and Scientific Research Publishing Inc. This work is licensed under the Creative Commons Attribution International License (CC BY 4.0).

http://creativecommons.org/licenses/by/4.0/

\begin{abstract}
Reversible phosphorylation and dephosphorylation play important roles in cell function and cell signal transduction. PPP2R5A (protein phosphatase 2 regulatory subunit $\mathrm{B}^{\prime}$ alpha) is responsible for specifically regulating the catalytic function, substrate specificity and intracellular localization of the tumor suppressor phosphatase PP2A (serine/threonine protein phosphatase 2A). Therefore, the abnormal expression and function of PPP2R5A may be related to canceration. The aim of this study was to reveal its role in the occurrence and development of hepatocellular carcinoma (HCC). It is hoped that the results of this study can provide guidance for the prevention and treatment of HCC. The results showed that PPP2R5A inhibited the proliferation and metastasis of HCC cells, and acted as a tumor suppressor in HCC cells, but it had no significant effect on cell cycle. Further research found that PPP2R5A exerted tumor suppressor efficacy by inhibiting the MAPK/AKT/WNT signaling pathway. Combined with analysis of clinical tissue samples and TCGA database, it was found that the expression of PPP2R5A in tumor tissues of Chinese HCC patients was down-regulated and significantly correlated with the progression-free survival (PFS) of HCC patients. On the contrary, PPP2R5A showed an up-regulation trend in HCC cases in TCGA database although its effect on PFS was the same with that in Chinese HCC patients.
\end{abstract}

\footnotetext{
*These authors contributed equally to this work.

"Corresponding author.
} 
Hepatitis B virus (HBV) infection is the main pathogenic factor of HCC in China. It was found that HBV infection reduced the content of PPP2R5A in cells. It was concluded that $\mathrm{HBV}$ inhibited the initiation of the protective mechanism mediated by PPP2R5A, making the occurrence and progress of HCC more "unimpeded". This conclusion will further reveal the role of PPP2R5A in HBV-induced and HBV-unrelated HCC, therefore, providing clues for the prevention and treatment of the two types of HCC, respectively.

\section{Keywords}

Hepatitis B Virus (HBV), Hepatocellular Carcinoma (HCC), Protein

Phosphatase 2 Regulatory Subunit B' Alpha (PPP2R5A), Serine/Threonine

Protein Phosphatase 2A (PP2A), Tumor Suppressor

\section{Introduction}

China has a high incidence of hepatocellular carcinoma (HCC), while the vast majority of HCC are associated with HBV infection [1] [2] [3]. In recent years, the anti-HCC effects of molecularly targeted drugs have attracted more and more attention and become one of the hot spots in anti-tumor research.

Reversible phosphorylation and dephosphorylation play important roles in the maintenance of cell homeostasis and the regulation of cell functions. About $50 \%$ of the serine/threonine dephosphorylation activity within cells is regulated by the serine/threonine protein phosphatase $2 \mathrm{~A}$ (PP2A). PP2A is the most abundant phosphatase in cells, and its expression accounts for $0.2 \%-1 \%$ of the total cell protein [4] [5] [6] [7]. Therefore, the expression level, substrate specificity, intracellular localization and enzyme activity of PP2A are closely related to cellular physiological state and pathological state. It is generally believed that PP2A is a tumor suppressor, which can negatively regulate cell division, inhibit protein synthesis and promote cell apoptosis, and is a potential target for tumor targeted therapy [8] [9].

Protein phosphatase 2 regulatory subunit B' alpha (PPP2R5A) is one of the regulatory subunits of $\mathrm{PP} 2 \mathrm{~A}$, which specifically regulates the phosphatase activity, substrate specificity and intracellular localization of PP2A. It has been reported that PPP2R5A is involved in the negative regulation of tumor-related proteins such as Bcl2 [10], MAPK [11], AKT [12], c-Myc [13] and $\beta$-Catenin [14], and abnormalities of PPP2R5A are often accompanied by occurrence of various diseases such as adenocarcinoma and epithelial cancer [15] [16] [17] [18]. It has been reported that lentiviral infection will increase the degradation of PPP2R5A to promote virus replication [19]. The analysis of HCC samples in the TCGA database found that PPP2R5A is upregulated in HCC patients, while the expression of PPP2R5A is significantly related to the prognosis of HCC. The HCC patients with high expression of PPP2R5A have a longer progression-free survival (PFS). The above clues suggested that PPP2R5A may exist as a tumor 
suppressor in the development of HCC. This study will explore the role and molecular mechanism of PPP2R5A in HCC.

\section{Material and Methods}

\subsection{Cell Lines and Cell Culture}

Human immortalized hepatocyte L02, WRL-68 and Chang liver cell lines, human HCC cell lines QGY-7701, Huh-7, SMMC-7721, Hep3B, HepG2, HepG2.2.15, PLC/PRF/5 and HCC-LM3 are provided by the Chinese Academy of Sciences Stem Cell Bank (Shanghai, China). Cells were cultured in DMEM (Gibco, USA) with $10 \% \mathrm{FBS}$ at $37^{\circ} \mathrm{C}$ and $5 \% \mathrm{CO}_{2}$.

\subsection{HCC Tissue Microarray and Immunohistochemistry (IHC) Evaluation}

The cancer and adjacent tissue samples from 110 cases of HCC patients were collected and made into HCC tissue microarray. The study has been approved by the Committee on Ethics of Medicine, Navy Military Medical University (Shanghai, China), and the patients have signed informed consents. The expression of PPP2R5A in HCC and adjacent tissues was detected by IHC. The AlgorithmS program of ImageScope software (Aperio) was used to calculate the "positive pixel" of the samples in tissue microarray. The calculation method of immunohistochemical score for each sample is $\log _{10}\left[255 / I_{\text {avg }}\right]$, where $I_{\text {avg }}$ represents the average intensity. The median of the cancer tissue IHC score was selected to distinguish between the high and low expression of PPP2R5A. Combined with the pathological and follow-up data, the correlations between PPP2R5A expression level and clinical indicators and survival period were analyzed.

\subsection{Western Blot}

Western blot was performed according to the conventional experimental methods. The antibodies used and the dilution ratio are shown in Supplementary Table S1.

\subsection{Plasmid Construction and Transfection}

The PPP2R5A knockdown vector pLKO.1-PPP2R5A shRNA (shRNA forward: 5'-CCGGCACTGAATGAACTGGTTGAGTCTCGAGACTCAACCAGTTCATT CAGTGTTTTTG-3'; shRNA reverse: 5'-AATTCAAAAACACTGAATGAACTGGTTGAGTCTCGAGACTCAACCAGTTCATTCAGTG-3') and the expression vector PLV-EF1 $\alpha$-PPP2R5A-EGFP were constructed. Lipo3000 liposome transfection reagent was used to transfect vectors into HCC cells. The total cell proteins were collected at $48 \mathrm{~h}$ after transfection for Western blot.

\subsection{CCK8 Assay}

The logarithmic growth phase cells were seeded in 96-well plates, the final con- 
centration was 5000 cells $/ 100 \mu \mathrm{L}$ medium ( 3 replicate wells per group). After being cultured in an incubator for $48 \mathrm{~h}$, cells of each well were added with $10 \mu \mathrm{L}$ of CCK8 reagent and incubated at $37^{\circ} \mathrm{C}$ for $2 \mathrm{~h}$ in dark, then placed in an enzyme-linked immunoassay instrument to detect $\mathrm{OD}_{450}$. The cell proliferation rate was calculated.

\subsection{Colony Formation Assay}

The logarithmic growth phase cells were seeded in 6-well plates, the final cell density was 1000 cells/well, and placed in an incubator for cultivation. After 14 days, cells were fixed with $4 \%$ formaldehyde, then stained with crystal violet for 15 - 20 min, washed with PBS for 3 - 4 times, and then photographed and statistically analyzed.

\subsection{Cell Cycle}

The cells in the logarithmic growth phase were collected and fixed with pre-cooled $75 \%$ alcohol, and placed at $4{ }^{\circ} \mathrm{C}$ overnight. The cells were centrifuged to remove alcohol, washed twice with PBS, added with $200 \mu \mathrm{L}$ of PI (BD Company, USA) staining solution to resuspend, then placed in dark to react for 30 minutes, and tested on the machine.

\subsection{Transwell assay}

The transwell chambers were coated with matrigel, and $5 \times 10^{4}$ cells were seeded into $200 \mu \mathrm{L}$ of serum-free DMEM in the upper chamber. Then $500 \mu \mathrm{L}$ of DMEM with $20 \%$ FBS were added to the lower chamber of transwell. The plate was placed in an incubator for static culture. After $48 \mathrm{~h}$, the upper chamber cells were wiped off, the migrated and invasive cells were stained with crystal violet for 15 minutes, and photographed and recorded under a microscope.

\subsection{Statistical Analysis}

The data were analyzed by SPSS 17.0 software package and expressed as mean \pm standard deviation (Mean $\pm \mathrm{SD}$ ). Analysis of variance (ANOVA) was used to analyze the data. $p<0.05$ indicates statistical significance. The statistical graphs were made with Graphpad Prism 5 software.

\section{Results}

\subsection{Low Expression of PPP2R5A in HCC Tissue Is Significantly Related to Poor Prognosis of Patients}

In order to explore the expression level of PPP2R5A in Chinese HCC patients, IHC detection was performed on tissue microarrays containing cancer and adjacent tissues from 110 HCC patients. The results showed that the expression of PPP2R5A in 77 cases (70\%) of cancer tissues was lower than that in the adjacent tissues, and that in 25 cases (22.7\%) did not change significantly. Its expression 
in 8 cases $(7.3 \%)$ of cancer tissue was higher than that in adjacent tissues (Figure 1(a), Figure 1(b)). The expression of PPP2R5A in each sample of tissue microarray was scored by ImageScope (Aperio) software. The results of statistical analysis showed that the expression score of PPP2R5A in HCC tissues was significantly lower than that in adjacent tissues, and the difference was statistically significant $(p<0.001)$ (Figure $1(c))$. Western Blot was used to detect the content of PPP2R5A in the total proteins of 12 pairs of HCC and adjacent tissues. It was found that the content of PPP2R5A protein in HCC tissues was lower than that in adjacent tissues (Figure $1(\mathrm{~d})$ ). The above results indicated that the expression of PPP2R5A in HCC tissues of Chinese patients is down-regulated. Among the 366 HCC samples in the TCGA database, about 24\% (87/366) of the tumor tissues showed high expression of PPP2R5A (Supplementary Figure S1) (http://www.cbioportal.org/), and others showed the same expression levels of PPP2R5A between HCC tissues and adjacent tissues.

After scoring HCC tissue microarray, the median score of cancer tissue (0.24) was selected as the criterion for distinguishing the high and low expression levels of PPP2R5A. Combined with pathological and follow-up data, the correlation between the expression level of PPP2R5A and the survival time of HCC patients was analyzed. The higher expression level of PPP2R5A corresponds to longer progression-free survival (PFS) and overall survival (OS) $(p<0.05)$ (Figure 1(e)). Analysis of samples from the TCGA database also showed that the high expression of PPP2R5A corresponds to a long PFS (Supplementary Figure S1).

In summary, PPP2R5A is generally down regulated in cancer tissues of Chinese HCC patients, and it is significantly positively correlated with the PFS of HCC patients.

\subsection{PPP2R5A Inhibits the Proliferation of HCC Cells}

PPP2R5A was knocked down and overexpressed in HCC QGY-7701 and Hep3B cells respectively. Western blot results showed that knockdown and overexpression of PPP2R5A were effective in both cell lines (Figure 2(a)). The results of colony formation assay showed that the number of colonies of HCC cells overexpressing PPP2R5A was significantly less than that of the control group, while the number of colonies of HCC cells that PPP2R5A were knocked down was significantly more than that of the control group (Figure 2(b)). CCK8 assay confirmed that the overexpression of PPP2R5A caused a decrease in the proliferation of QGY-7701 and Hep3B cells, while knockdown of PPP2R5A caused an increase in the proliferation of the two HCC cell sublines (Figure 2(c)).

Western Blot results showed that the overexpression of PPP2R5A resulted in the down-regulation of cell proliferation related indicators such as Bcl-2 and c-Myc. The knockdown of PPP2R5A yielded the opposite results (Figure 2(d), Figure 2(e)). The changes of protein molecules were consistent with the inhibition of cell proliferation by PPP2R5A. 
a

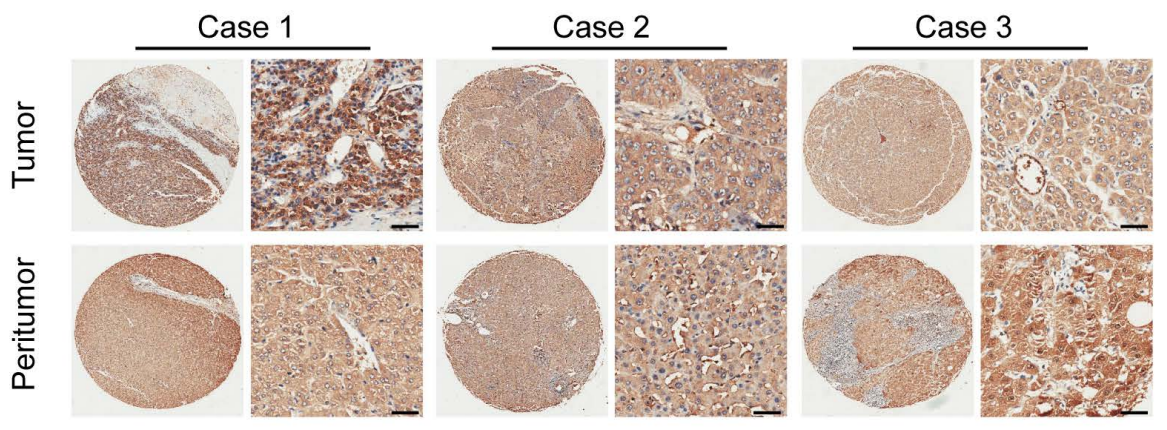

b

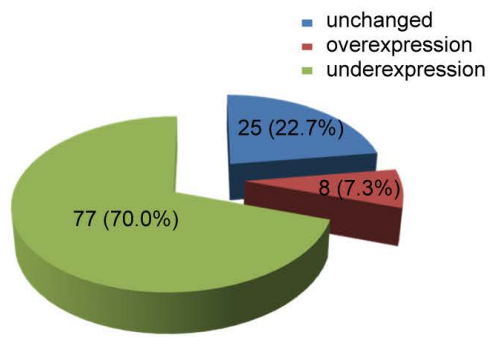

C

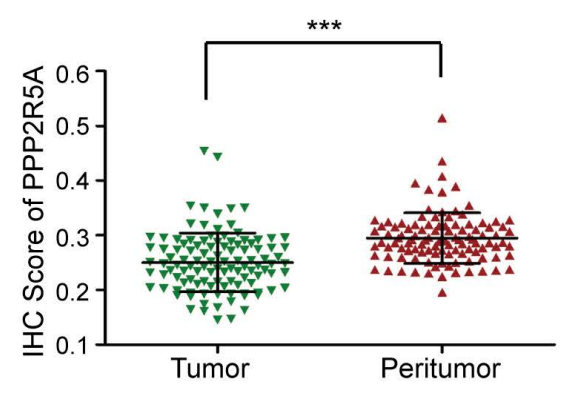

d

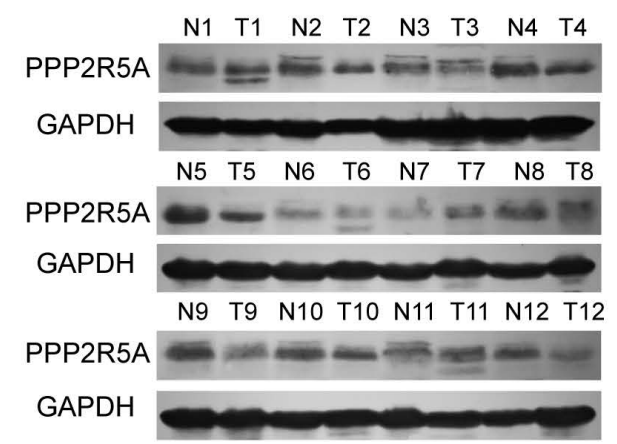

e

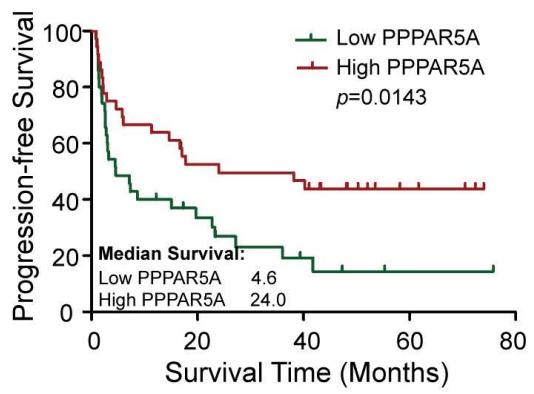

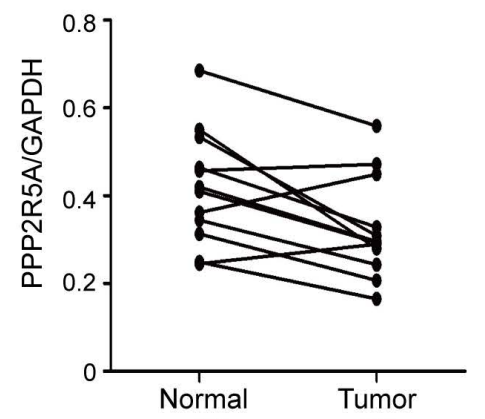

$(\mathrm{N})$

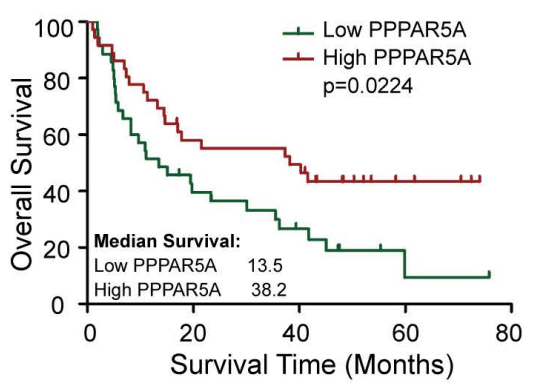

Figure 1. The low expression of PPP2R5A is significantly related to the poor prognosis of HCC. (a) IHC results showed that the higher, lower and comparable expression of PPP2R5A were found in HCC tissues compared with peritumor tissues in the tissue microarray. Case 1: tumor $>$ peritumor; Case 2: tumor $\approx$ peritumor; Case 3: tumor $<$ peritumor. Bar $=50 \mu \mathrm{m}$; (b) Statistical analysis of the expression of PPP2R5A in HCC tissues relative to the adjacent tissues; (c) IHC scores showed that the expression of PPP2R5A in HCC tissues is lower than that of adjacent tissues ( $\left.{ }^{* * *} p<0.001\right)$; (d) Western blot of PPP2R5A in the total proteins of 12 pairs of HCC and adjacent tissues and semi-quantitative statistical analysis; (e) Survival analysis showed that low expression of PPP2R5A was related to the poor prognosis of HCC patients. 
a

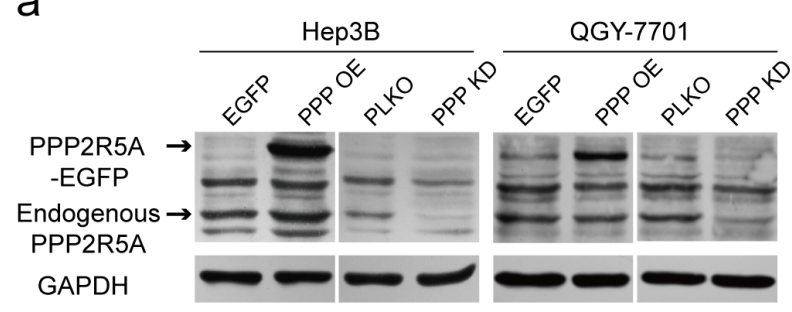

b

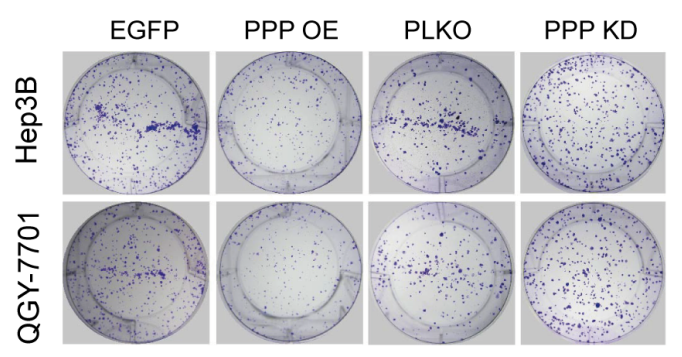

C

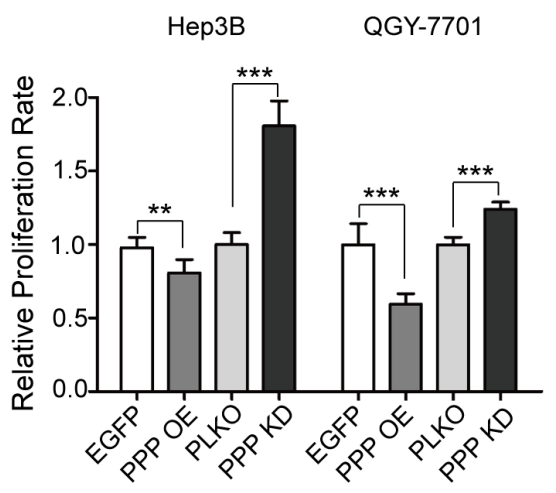

e

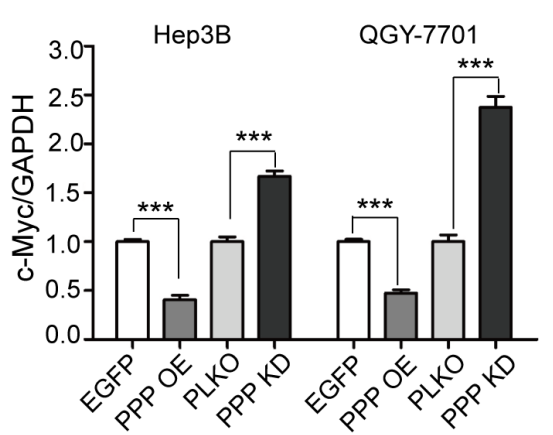

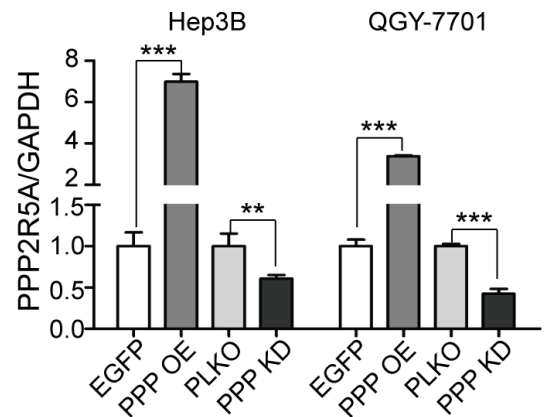

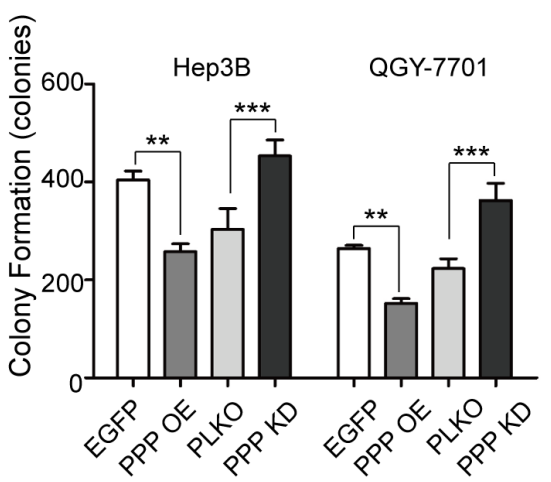

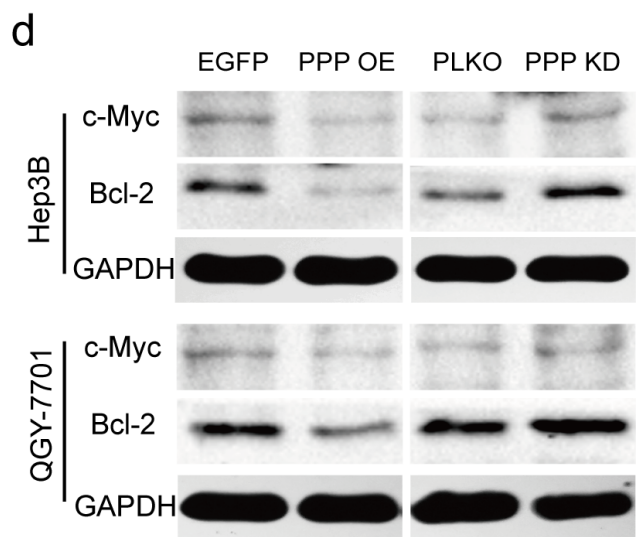

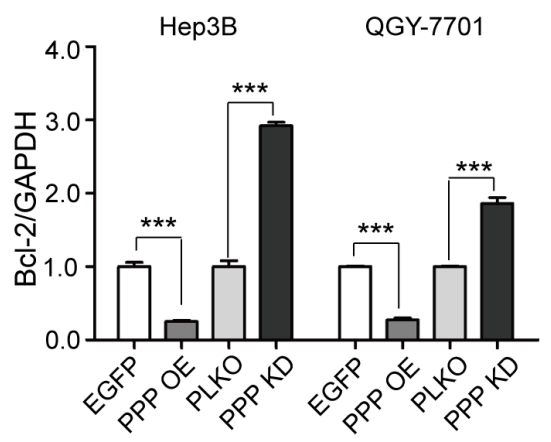

Figure 2. PPP2R5A inhibits the proliferation of HCC cells. (a) Western Blot to verify the overexpression and knockdown of PPP2R5A in Hep3B and QGY-7701 cells; (b) Colony formation assay to verify the effect of PPP2R5A on cell colony forming ability; (c) CCK8 assay to verify the effect of PPP2R5A on cell proliferation; (d), (e) Western Blot to detect the cell proliferation related indicators such as Bcl-2 and c-Myc and the semi-quantitative statistical analysis. $\left({ }^{* *} p<0.01\right.$; $\left.{ }^{* *} p<0.001\right)$. 


\subsection{PPP2R5A Inhibits the Metastasis of HCC Cells but Has No Effect on Cell Cycle}

The effects of PPP2R5A on cell cycle were detected by flow cytometry. The results showed that PPP2R5A had no significant effect on cell cycle (Figure 3(a)). The results of wound healing and transwell assays showed that the high expression of PPP2R5A inhibited the migration and invasion of HCC QGY-7701 and Hep3B cells, while the knockdown of PPP2R5A significantly promoted HCC cell metastasis (Figure 3(b), Figure 3(c)).

\subsection{HBV Infection Leads to the Down-Regulation of PPP2R5A Expression}

From the above results, it can be known that the expression of PPP2R5A in the cancer tissues from $70 \%$ of Chinese HCC patients is down-regulated and shows a certain tumor suppressor effect. Among the 366 HCC samples from TCGA database, about $24 \%$ of cancer tissues showed high expression of PPP2R5A (Supplementary Figure S1). Considering that there are great differences in the regional pathogenic factors of HCC, the vast majority of HCC patients from China are HBV positive, it is speculated that the down-regulation of PPP2R5A expression may be related to HBV infection.

In HCC and normal liver tissues, the expression of PPP2R5A in HBV-positive tissues is lower than that in HBV-negative tissues (Figure 4(a)). The results of HCC tissue microarray analysis showed that the expression of PPP2R5A was not significantly correlated with gender, age, AFP value and TNM staging of HCC patients, but was significantly correlated with the expression of HBsAg and pathological grade $(p<0.05)$ (Table 1). Western Blot found that the content of PPP2R5A in HepG2.2.15 ( $\mathrm{HBV}+)$ cells was lower than its parental HepG2 (HBV-) cells (Figure 4(b)), suggesting that the expression of PPP2R5A might be inhibited by HBV infection. Western Blot results showed that PPP2R5A was generally low expressed in HCC QGY-7701, PLC/PRF/5, and Hep3B cell lines that were integrated by HBV in cell genome (Figure 4(c)).

It is concluded from the above results that HBV infection down-regulates the content of PPP2R5A in hepatocytes. However, the specific mechanism through which HBV regulates the content of PPP2R5A remains unclear and needs to be further studied.

\subsection{PPP2R5A Exerts Tumor Suppressor Efficacy by Inhibiting the MAPK/AKT/WNT Signaling Pathway}

The MAPK and AKT signaling pathway play important roles in the process of tumor invasion and metastasis [20] [21]. The activation of WNT signaling pathway is also more common in cancer development [22] [23]. PPP2R5A is a regulatory subunit of intracellular phosphatase PP2A, responsible for the specific regulation of the enzymatic function, substrate specificity and intracellular localization of the tumor suppressor phosphatase PP2A, and likely to participate in the regulation of the above-mentioned signaling pathways. 
a
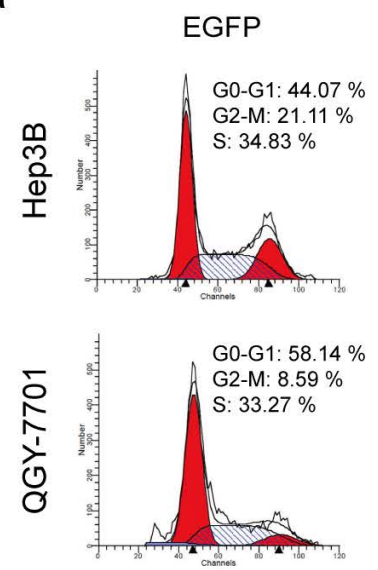

PPP OE
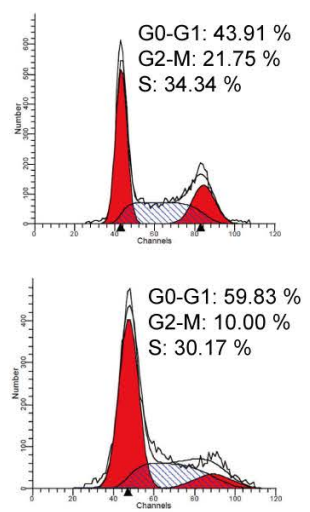

PLKO
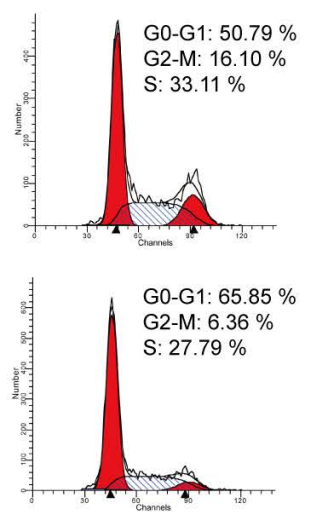

PPP KD
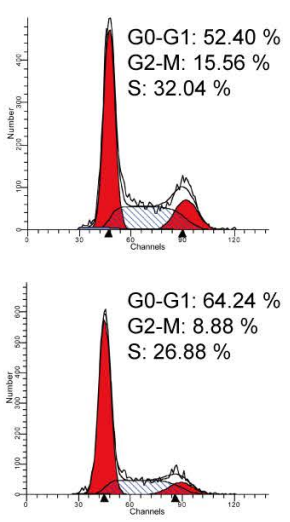

b
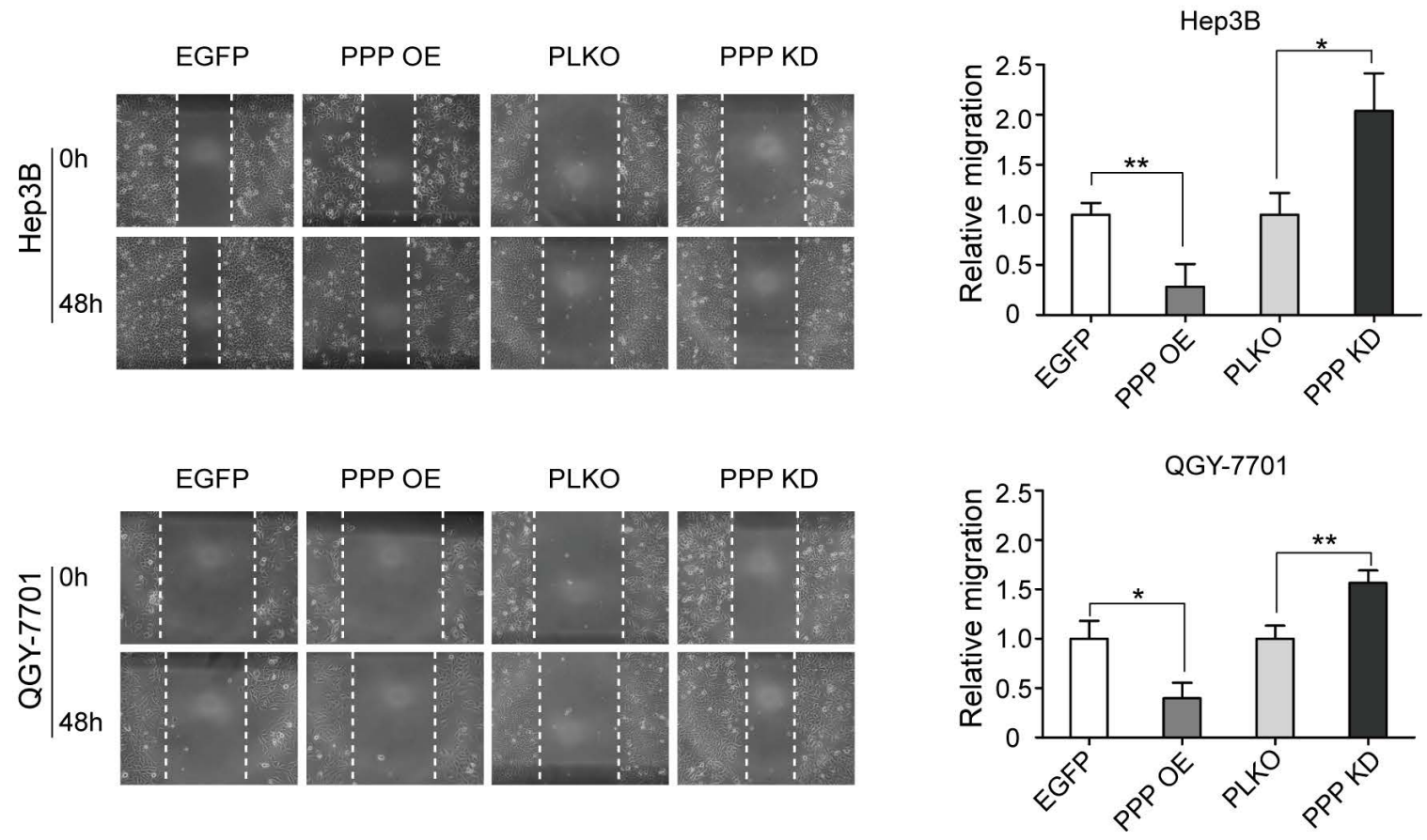

C
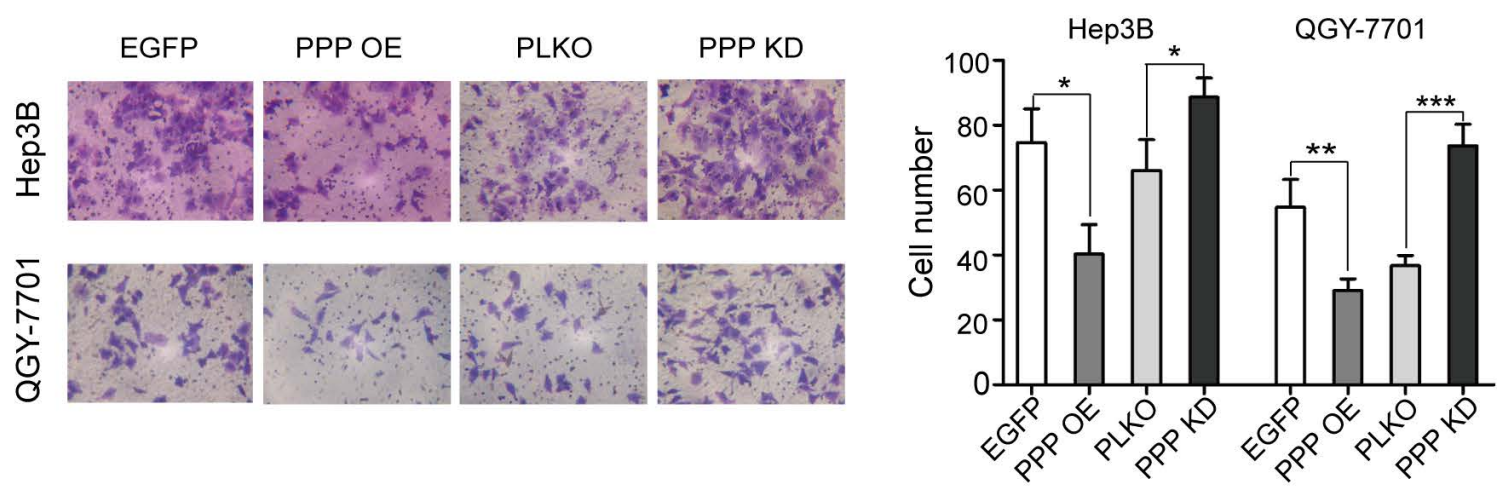

Figure 3. PPP2R5A inhibits the migration and invasion of HCC cells. (a) The effect of PPP2R5A on cell cycle; (b) Wound healing assay to detect the effect of PPP2R5A on cell migration, and the statistical analysis; (c) Transwell assay to detect the effect of PPP2R5A on cell invasion, and the statistical analysis. $\left({ }^{\star} p<0.05 ;{ }^{* *} p<0.01 ;{ }^{* *} p<0.001\right)$. 
a
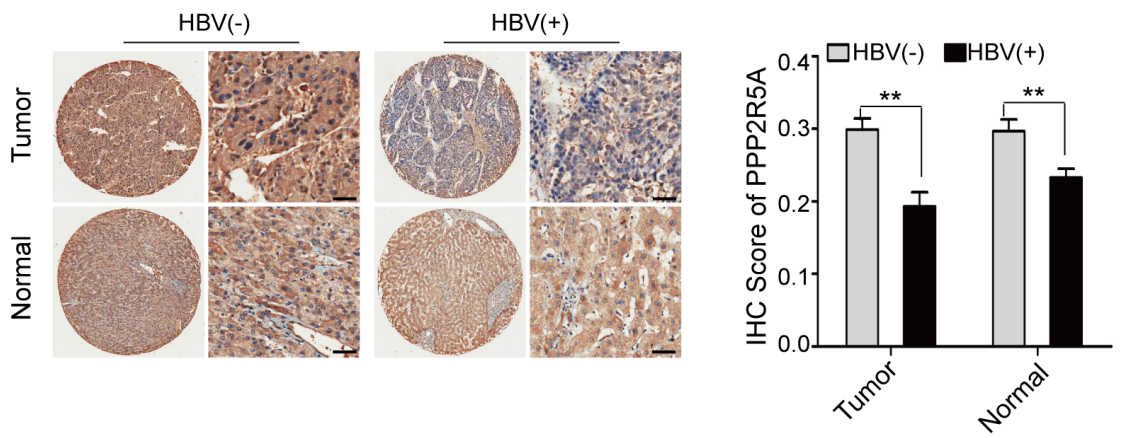

b

C
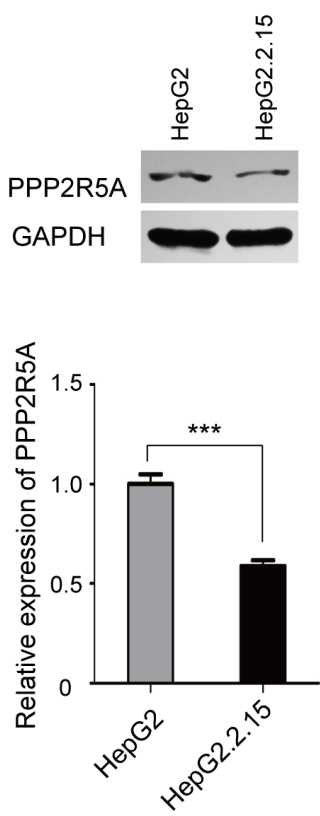
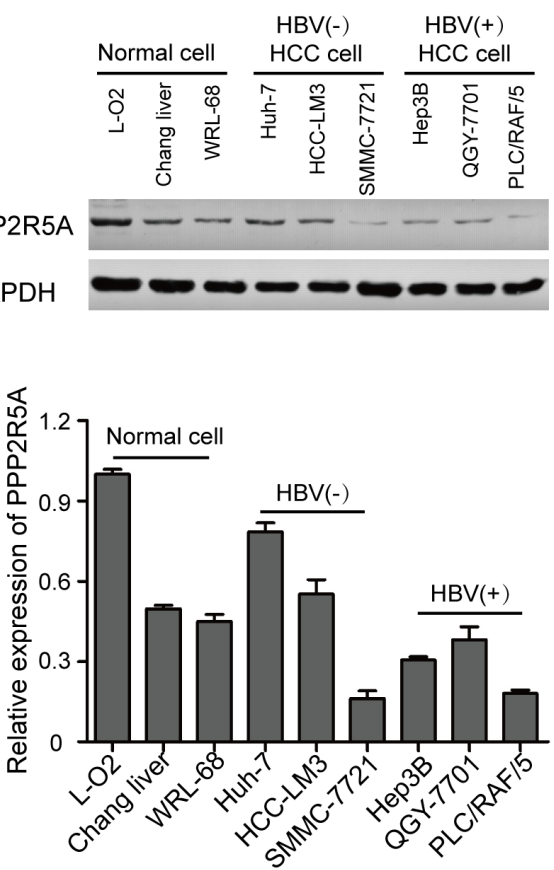

Figure 4. HBV infection leads to the down-regulation of PPP2R5A. (a) The differences in the expression of PPP2R5A in liver tissues of $\mathrm{HBV}(+)$ and $\mathrm{HBV}(-), \mathrm{Bar}=50 \mu \mathrm{m}$; (b) Western Blot of PPP2R5A in HepG2.2.15 HBV(+) and HepG2 HBV(-) HCC cells, and the bottom is the semi-quantitative statistical bar graph; (c) Western Blot of PPP2R5A in $\mathrm{HBV}(+)$ and HBV(-) HCC cells, and the semi-quantitative statistical bar graph is shown below. $\left.{ }^{* *} p<0.01 ;{ }^{* * *} p<0.001\right)$.

Western Blot was used to detect the phosphorylation levels of Raf, ERK and AKT in HCC cells after PPP2R5A knocked down and overexpressed. The results showed that the overexpression of PPP2R5A significantly reduced the phosphorylation levels of Raf, ERK and AKT, and down-regulated the protein level of $\beta$-Catenin in cells. While the knockdown of PPP2R5A obtained the opposite results. The $\mathrm{p}$-RAF, $\mathrm{p}$-ERK, and $\mathrm{p}$-AKT are all upstream factors that regulate $\beta$-Catenin. It is speculated that PPP2R5A inhibits the activation of the WNT pathway by inhibiting the MAPK and AKT signaling pathways, and downregulates $\beta$-Catenin, thereby exerting a tumor suppressor efficacy (Figure 5(a), Figure $5(\mathrm{~b}))$. 
a
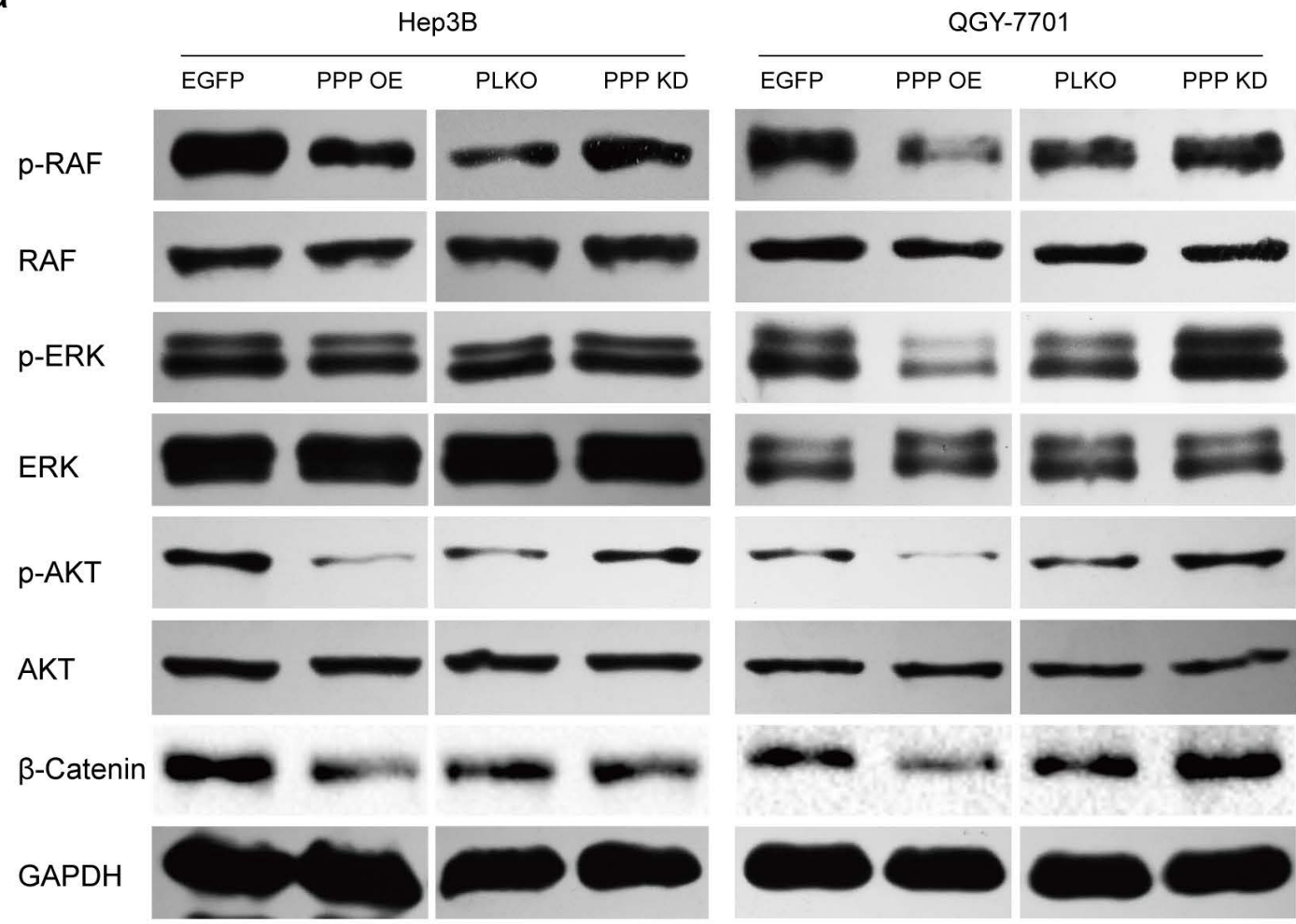

b
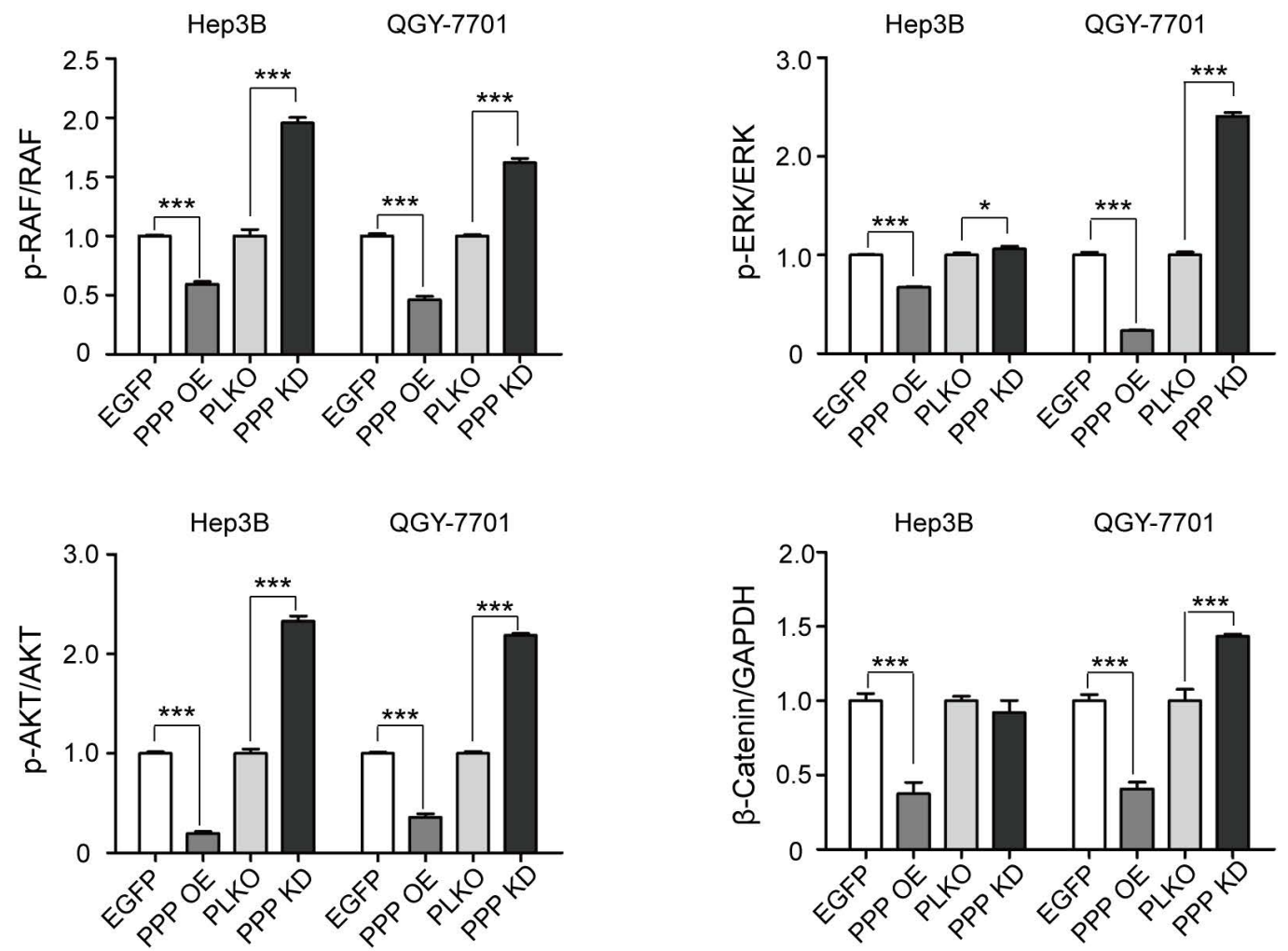

Figure 5. PPP2R5A exerts tumor suppressor efficacy by inhibiting the MAPK/AKT/WNT signaling pathway. (a) Western Blot to detect the influence of PPP2R5A on the related molecules of cell signaling pathways; (b) The semi-quantitative analysis of the relative changes of each molecule. $\left({ }^{*} p<0.05 ;{ }^{* * *} p<0.001\right)$. 
Table 1. Correlation analysis of PPP2R5A expression level and clinical data.

\begin{tabular}{|c|c|c|c|}
\hline Variables & Low $(n=55)$ & High $(\mathrm{n}=55)$ & p-value \\
\hline Gender, $\mathrm{x}(\%)$ & & & 0.567 \\
\hline Female & $8(14.5)$ & $6(10.9)$ & \\
\hline Male & $47(85.5)$ & $49(89.1)$ & \\
\hline Age, $x(\%)$ & & & 0.34 \\
\hline$\geq 50$ & $30(54.5)$ & $25(45.5)$ & \\
\hline$<50$ & $25(45.5)$ & $30(54.5)$ & \\
\hline HBsAg, x (\%) & & & 0.039 \\
\hline Negative & $3(5.5)$ & $10(18.2)$ & \\
\hline Positive & $52(94.5)$ & $45(81.8)$ & \\
\hline AFP, $x(\%)$ & & & 0.381 \\
\hline Negative, $<20 \mu \mathrm{g} / \mathrm{L}$ & $12(21.8)$ & $16(29.1)$ & \\
\hline Positive, $\geq 20 \mu \mathrm{g} / \mathrm{L}$ & $43(78.2)$ & $39(70.9)$ & \\
\hline TNM stage, $\mathrm{x}(\%)$ & & & 0.175 \\
\hline I and II & $29(56.4)$ & $36(61.8)$ & \\
\hline III and IV & $26(43.6)$ & $19(38.2)$ & \\
\hline Pathological grade, $\mathrm{x}(\%)$ & & & 0.045 \\
\hline I and II & $14(25.5)$ & $24(43.6)$ & \\
\hline III and IV & $41(74.5)$ & $31(56.4)$ & \\
\hline
\end{tabular}

\section{Discussion}

PPP2R5A has been reported to be involved in the regulation of signaling pathways of tumorigenesis [24]. Studies have shown that PP2A negatively regulated the cell anti-apoptotic factor Bcl2, PPP2R5A assisted PP2A in its functions and promoted the occurrence of apoptosis [11] [25]. Yamamoto et al. reported that PPP2R5A helped PP2A locate the important proto-oncoprotein $\beta$-Catenin in the WNT pathway by binding to the scaffold protein Axin, and promoted $\beta$-Catenin degradation [26]. Rodriguez et al. found that the lack of function of the PPP2R5A protein family led to the hyperfunction of the protein kinase B (AKT) family and promoted the activation of the WNT pathway and the occurrence of tumors [12] [26]. In addition, studies have shown that after inhibited the expression of PPP2R5A by microRNA-218, the WNT pathway would be significantly activated [14]. PPP2R5A can also assist PP2A to localize to c-Myc protein and promote the dephosphorylation and degradation of c-Myc protein [24]. These oncoproteins play important roles in the unlimited proliferation, infiltration and metastasis of tumor cells, and cell apoptosis resistance.

It is worth noting that some studies have found that viral infection could af- 
fect the expression of PPP2R5A. After treated with ceramide or double-stranded RNA, the eukaryotic translation initiation factor 2 alpha kinase 2 (PKR) of the cells activated and phosphorylated the 28th serine of PPP2R5A, which enhanced the inhibitory effect of PP2A on protein synthesis and induction of apoptosis, thereby inhibiting viral infection [27] [28]. However, hepatitis C virus (HCV) mRNA can promote Staufen1 to competitively inhibit the binding of viral mRNA and PKR, and block the autophosphorylation activation of PKR [29], which can lead to the decline of PPP2R5A expression and function. The Vif protein produced by lentiviral infection can recruit Cullin-5 (CUL5) E3 ubiquitin ligase to induce the ubiquitination and degradation of PPP2R5A [19] [30]. These examples demonstrated the antagonism of PPP2R5A and viral infection in the co-evolution of virus-host. However, the effect of HBV infection on PPP2R5A has not been reported yet.

Based on the above results, the molecular mechanism of PPP22R5A in the occurrence and development of HCC is speculated as follows (Figure 6): PPP2R5A exerts a tumor suppressor efficacy in hepatocytes. Under HBV-uninfected circumstances, when hepatocytes become cancerous, as one of the protective mechanisms, cells will up-regulate the expression of PPP2R5A. The high expression of PPP2R5A can inhibit the proliferation and metastasis of HCC cells. Therefore, the results of survival analysis showed that the higher the expression of PPP2R5A, the higher the PFS of HCC patients. But HBV is precisely the "nemesis" of PPP2R5A. When HCC patients are infected with HBV, HBV can down-regulate the content of PPP2R5A in cells, leading to a weakening of cellular self-protection mechanism. At this time, the expression of p-RAF, p-ERK,

a

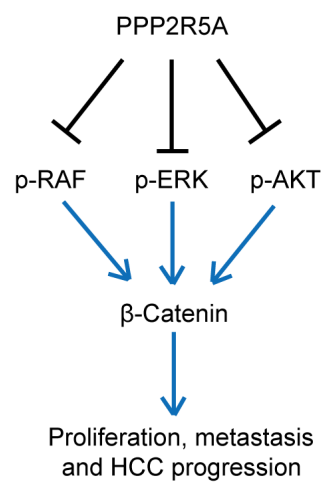

b

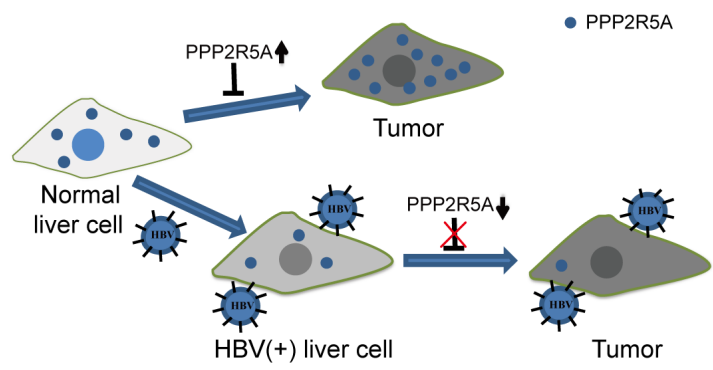

Figure 6. The molecular mechanism of HBV promoting the occurrence and development of HCC through PPP2R5A. (a) PPP2R5A inhibits the activation of WNT signaling pathway by inhibiting MAPK and AKT signaling pathways. (b) PPP2R5A acts as a tumor suppressor both in hepatocytes and HCC cells. Without HBV infection, when hepatocytes become cancerous, as one of the protective mechanisms, the cells will up-regulate the expression of PPP2R5A. The high expression of PPP2R5A can inhibit the proliferation and metastasis of HCC cells. When HCC patients are infected by HBV, HBV can down-regulate the content of PPP2R5A in cells. It leads to the reduction of PPP2R5A that exerts a tumor suppressor efficacy and the weakening of this cellular self-protection mechanism, thereby promoting the occurrence and development of HCC. 
and $\mathrm{p}$-AKT is up-regulated, and the activation of MAPK and AKT signaling pathways leads to an increase of $\beta$-Catenin, the key molecule of WNT signaling pathway, and an activation of WNT signaling pathway. The continuous activation of MAPK/AKT/WNT signaling pathways can promote cell proliferation and metastasis, thereby promoting the occurrence and development of HCC.

Combined with the fact that $85 \%-90 \%$ of HCC in China is closely related to $\mathrm{HBV}$ infection, it may be that HBV infection weakens the self-protection function of hepatocytes and increases the possibility of cell canceration.

Although the above data have confirmed that HBV infection can reduce the content of PPP2R5A, there are still many concrete problems for us to study further. Which protein or component of HBV is involved in the regulation of PPP2R5A? Is it the result of the host's immune response stimulated by HBV infection? Is it caused by the insertion of the HBV genes into the host genome? The answers to these questions are still unknown. The specific mechanism of HBV infection on PPP2R5A expression needs to be explored and confirmed by further proteomics analysis experiments.

\section{Conclusion}

PPP2R5A acted as a tumor suppressor in HCC cells. HBV inhibited the initiation of the protective mechanism mediated by PPP2R5A, making the occurrence and progress of HCC more "unimpeded". This conclusion will further reveal the molecular mechanism of HBV-induced HCC and provide clues for the prevention and treatment of HCC induced by HBV infection.

\section{Funding}

This work was supported by the National Key R\&D Program of China (2018 YFA0900902) and the National Natural Science Foundation of China (81702735 and 81773251).

\section{Ethics Statement}

The studies involving human participants were reviewed and approved by the Committee on Ethics of Medicine, Navy Military Medical University (Shanghai, China). The patients/participants provided their written informed consent to participate in this study.

\section{Compliance with Ethics Guidelines}

Xuejing Lin, Ziming Mao, Qin Zhang, Lei Chen, Haihua Qian, Chunying Liu and Changqing Su declare that they have no conflict of interest or financial conflicts to disclose.

\section{Conflicts of Interest}

The authors declare no conflicts of interest regarding the publication of this paper. 


\section{References}

[1] Xie, D.Y., Ren, Z.G., Zhou, J., Fan, J. and Gao, Q. (2020) Chinese Clinical Guidelines for the Management of Hepatocellular Carcinoma: Updates and Insights. Hepatobiliary Surgery and Nutrition, 9, 452-463. https://doi.org/10.21037/hbsn-20-480

[2] Jia, L., Gao, Y., He, Y., Hooper, J.D. and Yang, P. (2020) HBV Induced Hepatocellular Carcinoma and Related Potential Immunotherapy. Pharmacological Research, 159, Article ID: 104992. https://doi.org/10.1016/j.phrs.2020.104992

[3] Liu, S., Zhou, B., Valdes, J.D., Sun, J. and Guo, H. (2019) Serum Hepatitis B Virus RNA: A New Potential Biomarker for Chronic Hepatitis B Virus Infection. Hepatology, 69, 1816-1827. https://doi.org/10.1002/hep.30325

[4] Sangodkar, J., Farrington, C.C., McClinch, K., Galsky, M.D., Kastrinsky, D.B. and Narla, G. (2016) All Roads Lead to PP2A: Exploiting the Therapeutic Potential of This Phosphatase. The FEBS Journal, 283, 1004-1024. https://doi.org/10.1111/febs.13573

[5] Virshup, D.M. and Shenolikar, S. (2009) From Promiscuity to Precision: Protein Phosphatases Get a Makeover. Molecular Cell, 33, 537-545. https://doi.org/10.1016/j.molcel.2009.02.015

[6] Wlodarchak, N. and Xing, Y. (2016) PP2A as a Master Regulator of the Cell Cycle. Critical Reviews in Biochemistry and Molecular Biology, 51, 162-184. https://doi.org/10.3109/10409238.2016.1143913

[7] Fowle, H., Zhao, Z. and Graña, X. (2019) PP2A Holoenzymes, Substrate Specificity Driving Cellular Functions and Deregulation in Cancer. Advances in Cancer Research, 144, 55-93. https://doi.org/10.1016/bs.acr.2019.03.009

[8] Clark, A.R. and Ohlmeyer, M. (2019) Protein Phosphatase 2A as a Therapeutic Target in Inflammation and Neurodegeneration. Pharmacology \& Therapeutics, 201, 181-201. https://doi.org/10.1016/j.pharmthera.2019.05.016

[9] Bao, Y., Oguz, G., Lee, W.C., Lee, P.L., Ghosh, K., Li, J., et al. (2020) EZH2-Mediated PP2A Inactivation Confers Resistance to HER2-Targeted Breast Cancer Therapy. Nature Communications, 11, Article No. 5878. https://doi.org/10.1038/s41467-020-19704-x

[10] Liu, Z., Yoshimi, A., Wang, J., Cho, H., Lee, S.C.-W., Ki, M., et al. (2020) Mutations in the RNA Splicing Factor SF3B1 Promote Tumorigenesis through MYC Stabilization. Cancer Discovery, 10, 806-821. https://doi.org/10.1158/2159-8290.CD-19-1330

[11] Silverstein, A.M., Barrow, C.A., Davis, A.J. and Mumby, M.C. (2002) Actions of PP2A on the MAP Kinase Pathway and Apoptosis Are Mediated by Distinct Regulatory Subunits. Proceedings of the National Academy of Sciences of the United States of America, 99, 4221-4226. https://doi.org/10.1073/pnas.072071699

[12] Rodriguez Pino, M., Castillo, B., Kim, B. and Kim, L.W. (2015) PP2A/B56 and GSK3/Ras Suppress PKB Activity during Dictyostelium chemotaxis. Molecular Biology of the Cell, 26, 4347-4357. https://doi.org/10.1091/mbc.E14-06-1130

[13] Kauko, O. and Westermarck, J. (2018) Non-Genomic Mechanisms of Protein Phosphatase 2A (PP2A) Regulation in Cancer. The International Journal of Biochemistry \& Cell Biology, 96, 157-164. https://doi.org/10.1016/j.biocel.2018.01.005

[14] Zhuang, Z., Hu, F., Hu, J., Wang, C., Hou, J., Yu, Z., et al. (2017) MicroRNA-218 Promotes Cisplatin Resistance in Oral Cancer via the PPP2R5A/Wnt Signaling Pathway. Oncology Reports, 38, 2051-2061. https://doi.org/10.3892/or.2017.5899

[15] Forbes, S.A., Tang, G., Bindal, N., Bamford, S., Dawson, E., Cole, C., et al. (2010) COSMIC (The Catalogue of Somatic Mutations in Cancer): A Resource to Investi- 
gate Acquired Mutations in Human Cancer. Nucleic Acids Research, 38, D652-D657. https://doi.org/10.1093/nar/gkp995

[16] Kirchhefer, U., Heinick, A., König, S., Kristensen, T., Müller, F.U., Seidl, M.D., et al. (2014) Protein Phosphatase 2A Is Regulated by Protein Kinase C $\alpha$ (PKC $\alpha)$-Dependent Phosphorylation of Its Targeting Subunit B56 $\alpha$ at Ser41. Journal of Biological Chemistry, 289, 163-176. https://doi.org/10.1074/jbc.M113.507996

[17] Giannakis, M., Hodis, E., Mu, J.X., Yamauchi, M., Rosenbluh, J., Cibulskis, K., et al. (2014) RNF43 Is Frequently Mutated in Colorectal and Endometrial Cancers. Nature Genetics, 46, 1264-1266. https://doi.org/10.1038/ng.3127

[18] DeGrande, S.T., Little, S.C., Nixon, D.J., Wright, P., Snyder, J., Dun, W., et al. (2013) Molecular Mechanisms Underlying Cardiac Protein Phosphatase 2A Regulation in Heart. Journal of Biological Chemistry, 288, 1032-1046.

https://doi.org/10.1074/jbc.M112.426957

[19] Greenwood, E.J., Matheson, N.J., Wals, K., van den Boomen, D.J., Antrobus, R., Williamson, J.C., et al. (2016) Temporal Proteomic Analysis of HIV Infection Reveals Remodelling of the Host Phosphoproteome by Lentiviral Vif Variants. Elife, 5, e18296. https://doi.org/10.7554/eLife.18296

[20] Liu, J.F., Chen, P.C., Chang, T.M. and Hou, C.H. (2020) Monocyte Chemoattractant Protein-1 Promotes Cancer Cell Migration via c-Raf/MAPK/AP-1 Pathway and MMP-9 Production in Osteosarcoma. Journal of Experimental \& Clinical Cancer Research, 39, Article No. 254. https://doi.org/10.1186/s13046-020-01756-y

[21] Wu, S., Chen, M., Huang, J., Zhang, F., Lv, Z., Jia, Y., et al. (2020) ORAI2 Promotes Gastric Cancer Tumorigenicity and Metastasis through PI3K/Akt Signaling and MAPK-Dependent Focal Adhesion Disassembly. Cancer Research, 81, 986-1000. https://doi.org/10.1158/0008-5472.CAN-20-0049

[22] Zuo, Q., He, J., Zhang, S., Wang, H., Jin, G., Jin, H., et al. (2021) PPAR $\gamma$ Coactivator-1a Suppresses Metastasis of Hepatocellular Carcinoma by Inhibiting Warburg Effect by PPAR $\gamma$-Dependent WNT/ $\beta$-Catenin/Pyruvate Dehydrogenase Kinase Isozyme 1 Axis. Hepatology, 73, 644-660. https://doi.org/10.1002/hep.31280

[23] Pan, J., Fang, S., Tian, H., Zhou, C., Zhao, X., Tian, H., et al. (2020) lncRNA $\mathrm{JPX} / \mathrm{miR}-33 \mathrm{a}-5 \mathrm{p} /$ Twist1 axis Regulates Tumorigenesis and Metastasis of Lung Cancer by Activating Wnt/ $\beta$-Catenin Signaling. Molecular Cancer, 19, Article No. 9. https://doi.org/10.1186/s12943-020-1133-9

[24] Arnold, H.K. and Sears, R.C. (2008) A Tumor Suppressor Role for PP2A-B56alpha through Negative Regulation of c-Myc and Other Key Oncoproteins. Cancer and Metastasis Reviews, 27, 147-158. https://doi.org/10.1007/s10555-008-9128-9

[25] Lee, N.S., Veeranki, S., Kim, B. and Kim, L. (2008) The Function of PP2A/B56 in Non-Metazoan Multicellular Development. Differentiation, 76, 1104-1110. https://doi.org/10.1111/j.1432-0436.2008.00301.x

[26] Yamamoto, H., Hinoi, T., Michiue, T., Fukui, A., Usui, H., Janssens, V., et al. (2001) Inhibition of the Wnt Signaling Pathway by the PR61 Subunit of Protein Phosphatase 2A. Journal of Biological Chemistry, 276, 26875-26882.

https://doi.org/10.1074/jbc.M100443200

[27] Ruvolo, P.P., Clark, W., Mumby, M., Gao, F. and May, W.S. (2002) A Functional role for the B56 Alpha-Subunit of Protein Phosphatase 2A in Ceramide-Mediated Regulation of Bcl2 Phosphorylation Status and Function. Journal of Biological Chemistry, 277, 22847-22852. https://doi.org/10.1074/jbc.M201830200

[28] Xu, Z. and Williams, B.R. (2000) The B56a Regulatory Subunit of Protein Phosphatase 2A Is a Target for Regulation by Double-Stranded RNA-Dependent Protein 
Kinase PKR. Molecular and Cellular Biology, 20, 5285-5299.

https://doi.org/10.1128/MCB.20.14.5285-5299.2000

[29] Dixit, U., Pandey, A.K., Mishra, P., Sengupta, A. and Pandey, V.N. (2016) Staufen1 Promotes HCV Replication by Inhibiting Protein Kinase R and Transporting Viral RNA to the Site of Translation and Replication in the Cells. Nucleic Acids Research, 44, 5271-5287. https://doi.org/10.1093/nar/gkw312

[30] Malim, M.H. and Bieniasz, P.D. (2012) HIV Restriction Factors and Mechanisms of Evasion. Cold Spring Harbor Perspectives in Medicine, 2, Article ID: a006940. https://doi.org/10.1101/cshperspect.a006940 


\section{Supplementary Material}

Table S1. Antibody information.

\begin{tabular}{cc}
\hline Antibody name & Product information \\
\hline Anti-PPP2R5A antibody & ab89621, Abcam, UK, dilution 1:1000 \\
Anti- $\beta$-Catenin antibody & 8480, CST, USA, dilution 1:1000 \\
Anti-c-Myc antibody & 9402, CST, USA, dilution 1:1000 \\
Anti-Bcl2 antibody & 2872, CST, USA, dilution 1:1000 \\
Anti-Raf antibody & 53745, CST, USA, dilution 1:1000 \\
Anti-p-Raf antibody & 9421, CST, USA, dilution 1:1000 \\
Anti-ERK antibody & 4695, CST, USA, dilution 1:1000 \\
Anti-p-ERK antibody & 4370, CST, USA, dilution 1:1000 \\
Anti-AKT antibody & 4691, CST, USA, dilution 1:1000 \\
Anti-p-AKT antibody & 4060, CST, USA, dilution 1:1000 \\
Anti-GAPDH antibody & KC5G5, Aksomics, CN, dilution 1:5000
\end{tabular}
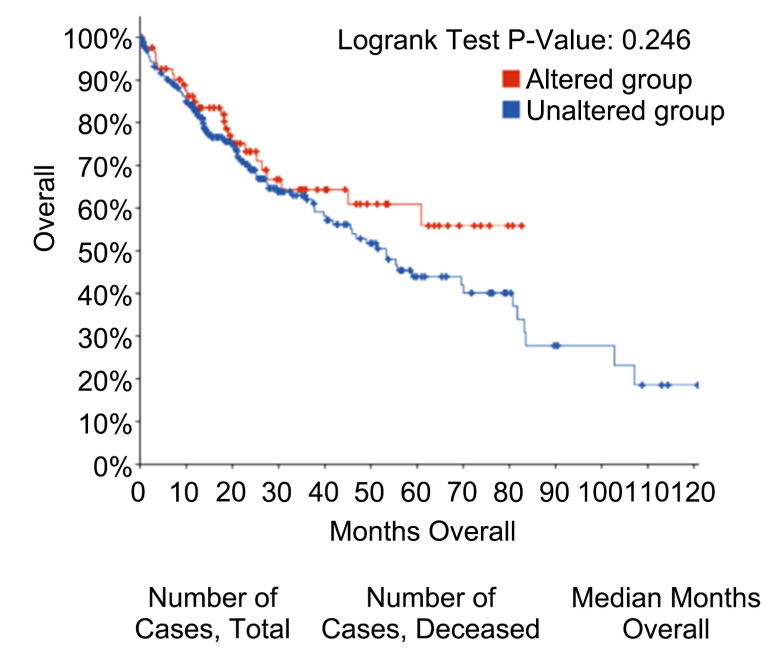

\section{Altered}

group

Unaltered group

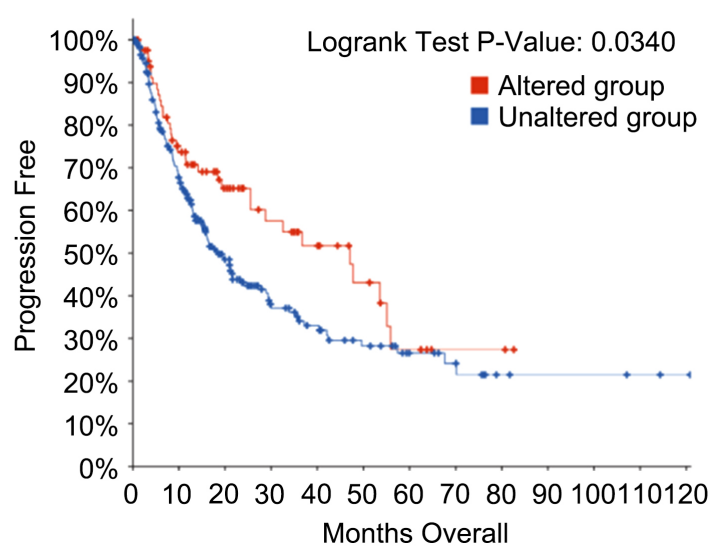

$\begin{array}{lccc} & \begin{array}{c}\text { Number of } \\ \text { Cases, Total }\end{array} & \begin{array}{c}\text { Number of } \\ \text { Cases, Progressed }\end{array} & \begin{array}{c}\text { Median Months } \\ \text { Progression Free }\end{array} \\ \begin{array}{l}\text { Altered } \\ \text { group }\end{array} & 87 & 25 & 47.08 \\ \begin{array}{l}\text { Unaltered } \\ \text { group }\end{array} & 278 & 42 & 18.61\end{array}$

(b)

Figure S1. Survival analysis of PPP2R5A expression in HCC patients from TCGA database (http://www.cbioportal.org/). (a) Analysis of overall survival ( $p=0.246)$. (b) Analysis of progression-free survival $(p<0.05)$. 